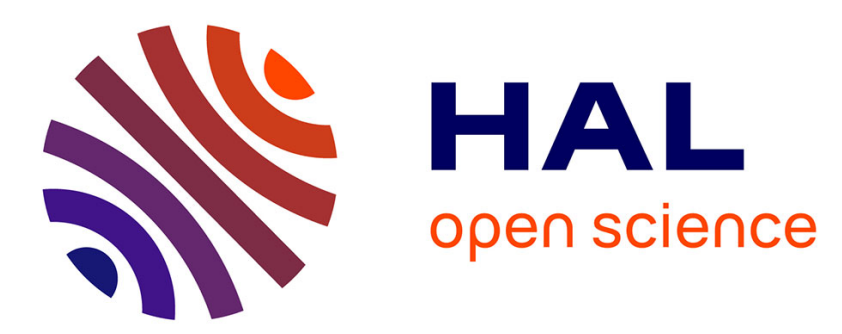

\title{
Dissociation des molécules H2 dans la décharge HF d'une source d'atomes polarisés
}

\author{
Michel Desaintfuscien
}

\section{To cite this version:}

Michel Desaintfuscien. Dissociation des molécules H2 dans la décharge HF d'une source d'atomes polarisés. Revue de Physique Appliquée, 1967, 2 (4), pp.235-238. 10.1051/rphysap:0196700204023500 . jpa-00242797

\section{HAL Id: jpa-00242797 https://hal.science/jpa-00242797}

Submitted on 1 Jan 1967

HAL is a multi-disciplinary open access archive for the deposit and dissemination of scientific research documents, whether they are published or not. The documents may come from teaching and research institutions in France or abroad, or from public or private research centers.
L'archive ouverte pluridisciplinaire HAL, est destinée au dépôt et à la diffusion de documents scientifiques de niveau recherche, publiés ou non, émanant des établissements d'enseignement et de recherche français ou étrangers, des laboratoires publics ou privés. 


\title{
DISSOGIATION DES MOLÉGULES $\mathrm{H}_{2}$ DANS LA DÉGHARGE HF D'UNE SOURGE D'ATOMES POLARISÉS
}

\author{
Par Mighel DESAINTFUSGIEN, \\ Institut d'Électronique Fondamentale, Faculté des Sciences, Bâtiment 220, 9I-Orsay, France.
}

Résumé. - On établit une expression de la concentration en atomes dans le jet issu de la décharge. L'influence de divers facteurs (pression dans la source, puissance et fréquence de l'excitation HF) est mise en évidence.

On étudie ensuite expérimentalement cette source. On trouve, selon les conditions, un pourcentage d'atomes dans le jet compris entre 50 et $65 \%$. L'étude expérimentale repose sur la relation entre l'intensité axiale du jet et le flux atomique focalisé à travers un diaphragme grâce à un aimant hexapolaire dans le fonctionnement d'une horloge à hydrogène alimentée par cette source.

Abstract. - We establish a formula giving the atomic concentration of the beam provided by the discharge.

We show the influence of different parameters (pressure of the source, power and frequency of the R.F. discharge). Depending on the conditions, the experimental study of this source gives a rate of dissociation varying between 50 and 65 per cent.

This study is based upon the relation between the axial intensity of the beam and the atomic flux focused by an hexapolar magnet in a hydrogen maser.

Introduction. - Cette source était destinée à une horloge à hydrogène. La décharge est excitée dans un tube en verre pyrex (fig. 1) par couplage inductif

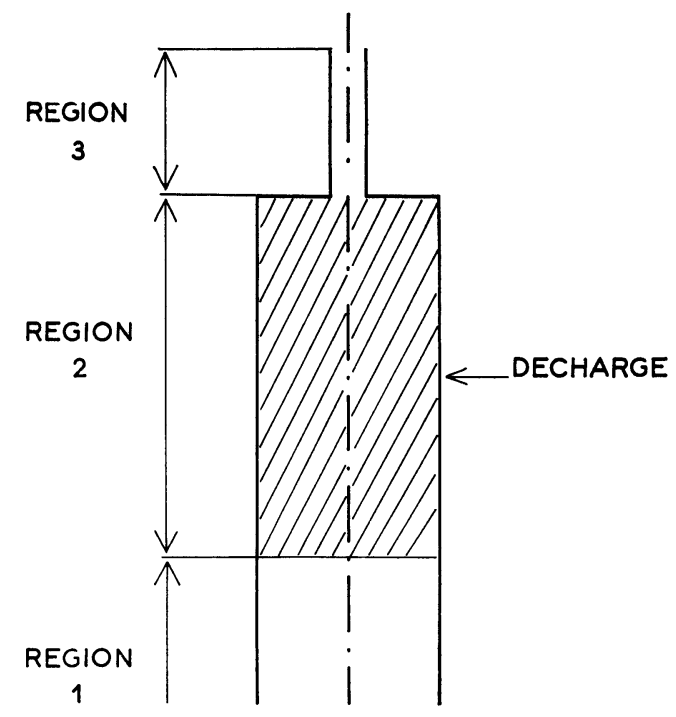

FIG. 1. - Schéma de la source.

avec un solénoïde coaxial au tube. Les dimensions transversales sont de l'ordre du centimètre. La pression du gaz est fixée, selon les besoins, entre quelque $10^{-2}$ et quelque $10^{-1}$ torr. La puissance dissipée est de quelques dizaines de watts, pour une fréquence d'excitation de $150 \mathrm{MHz}$ ou plus.

Les caractéristiques de la source. (voir les résultats $\mathrm{du} \S \mathrm{V})$ sont analogues à celles d'une source de protons polarisés de faible intensité [1]. Des sources plus intenses [2], [3], destinées à alimenter des accélérateurs, fournissent un flux atomique 100 fois plus important.

\section{Equation de diffusion de l'hydrogène atomique.} - On étudie l'équilibre qui existe, dans la décharge, entre les atomes qui apparaissent dans un élément de volume par réaction de dissociation, ceux qui en disparaissent à la suite d'une recombinaison et ceux qui y entrent ou en sortent, soit par diffusion, soit du fait de l'écoulement de l'ensemble du gaz.

La production des atomes a lieu presque uniquement par l'intermédiaire de la réaction

$$
H_{2}+\mathrm{e} \rightarrow 2 \mathrm{H}+\mathrm{e} \text {. }
$$

La fréquence $P$ de cette réaction est caractérisée par le facteur $Q$ tel que

$$
P=n_{2} n_{\mathrm{e}} Q
$$

où $n_{2}$ est la densité en volume des molécules $H_{2}$ et $n_{\mathrm{e}}$ celle des électrons. Les valeurs de $Q$ sont données dans la référence [4] en fonction de l'énergie électronique moyenne $\bar{w}$. Dans notre cas, $\bar{w}$ varie entre 3 et $6 \mathrm{eV}$, selon les conditions. Il apparaît donc dans la décharge $2 n_{2} n_{\mathrm{e}} Q$ atomes par seconde. 
La disparition des atomes par recombinaison a lieu dans le volume de la décharge et sur les parois qui la limitent.

Pour simplifier la résolution de l'équation de diffusion, on remplace les pertes sur les parois par des pertes globalement équivalentes qui seraient réparties dans le volume, ce qui est surtout justifié pour les plus faibles pressions [5].

Si $\rho_{0}$ est le coefficient de recombinaison de surface [6], $\bar{v}_{1}$ la vitesse moyenne des atomes, $n_{1}$ leur densité volumique et $R$ le rayon de l'appareil, on trouve que ces pertes sont égales à : $\rho_{0} \frac{\bar{v}_{1} n_{1}}{2 R}$, par unité de volume et de temps.

Les pertes qui se produisent effectivement dans le volume sont beaucoup moins importantes que celles qui se produisent sur les parois (elles ne peuvent avoir lieu qu'à l'occasion d'un choc triple) [7]. Elles sont caractérisées par une durée de vie $T_{1}$ des atomes qui est inversement proportionnelle à la concentration $\frac{n_{1}}{n_{1}+n_{2}}$ des atomes. On majore la correction intro-

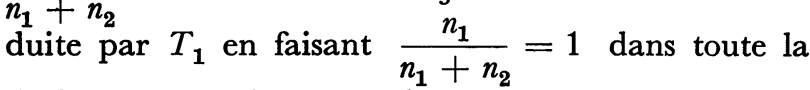
décharge, ce qui permet de conserver la linéarité de l'équation de diffusion.

En tenant compte enfin de l'écoulement de l'ensemble du gaz, de vitesse $u$, qui introduit une variation de la densité $n_{1}$ égale à $-\frac{\mathrm{d} n_{1}}{\mathrm{~d} x} u$ par unité de volume et de temps, et de la diffusion des atomes, qui introduit la variation $D \frac{\mathrm{d}^{2} n_{1}}{\mathrm{~d} x^{2}}$ de la densité $n_{1}$, si $D$ est le coeffi- cient de diffusion des atomes [8], on est conduit à l'équation :

$\frac{\mathrm{d}^{2} n_{1}}{\mathrm{~d} x^{2}}-\frac{u}{D} \frac{\mathrm{d} n_{1}}{\mathrm{~d} x}-\frac{1}{D}\left(\frac{\rho_{\mathrm{o}} \bar{v}_{1}}{2 R}+\frac{1}{T_{1}}\right) n_{1}=-2 n_{\mathrm{e}} n_{2} \frac{Q}{D}$

qui peut s'écrire :

$\frac{\mathrm{d}^{2} n_{1}}{\mathrm{~d} x^{2}}-\frac{u}{D} \frac{\mathrm{d} n_{1}}{\mathrm{~d} x}-\frac{1}{D}\left(\frac{\rho_{\mathrm{o}} v_{1}}{2 R}+\frac{1}{T_{1}}+2 n_{\mathrm{e}} Q\right) n_{1}$

$$
=-2 n_{\mathrm{e}} n \frac{Q}{D}
$$

en posant $n=n_{1}+n_{2}$.

Cette équation est générale en ce sens qu'on peut l'appliquer dans toutes les régions de l'appareil, à condition de faire $Q=0$ en dehors de la zone de décharge proprement dite.

II. Résolution de l'équation de diffusion. - La décharge se produit dans la région qui précède immédiatement le canal d'évacuation du gaz (fig. 1). Il y a donc les 3 régions $1,2,3$ définies sur la figure 1 . La décharge se produit dans la région 2 et $Q=0$ dans les deux autres régions.

Les conditions de continuité de $n_{1}(x)$ et de $\frac{\mathrm{d} n_{1}(x)}{d x}$ à chacune des surfaces de séparation et le fait que $n_{1}(x)$ doit tendre vers 0 dans les régions 1 et 3 quand $x$ devient infiniment grand permettent de déterminer les constantes d'intégration dans chacune des régions.

On en déduit l'expression suivante du taux de dissociation $m$ à la sortie du tube : $m=\frac{n_{1}}{n_{1}+n_{2}}$.

avec

$$
m=\frac{2 n_{\mathrm{e}} Q}{\rho_{\mathrm{c}} \frac{\bar{v}_{1}}{2 R}+\frac{1}{T_{1}}+2 n_{\mathrm{e}} Q} \alpha_{2} \mathrm{e}^{-\left(\beta_{\mathrm{s}}-\frac{\lambda \mathrm{u}}{2 D}\right) l^{\prime}} \frac{\operatorname{sh} \alpha_{2} l+\beta_{1}\left(\operatorname{ch} \alpha_{2} l-1\right)}{A \operatorname{ch} \alpha_{2} l+B \operatorname{sh} \alpha_{2} l}
$$

où

$$
A=\alpha_{2}\left(\beta_{1}+\beta_{3}-\frac{\lambda u}{2 D}\right) \quad B=\alpha_{2}^{2}+\beta_{1}\left(\beta_{3}-\frac{\lambda u}{2 D}\right)
$$

$$
\begin{gathered}
\alpha_{2}^{2}=\frac{u^{2}}{4 D^{2}}+\frac{1}{D}\left(\rho_{\mathrm{c}} \frac{\bar{v}_{1}}{2 R}+\frac{1}{T_{1}}+2 n_{\mathrm{e}} Q\right) \\
\beta_{1}^{2}=\frac{1}{D}\left(\rho_{\mathrm{c}} \frac{\bar{v}_{1}}{2 R}+\frac{1}{T_{1}}\right)+\frac{u^{2}}{4 D^{2}} \quad \beta_{3}^{2}=\frac{1}{D}\left(\rho_{\mathrm{c}} \frac{\bar{v}_{1}}{2 R}+\frac{1}{T_{1}}\right)+\frac{\lambda^{2} u^{2}}{4 D^{2}}
\end{gathered}
$$

$\lambda$ est le rapport entre la section du tube de décharge et celle du tube d'évacuation (région 3).

Ce résultat fait intervenir le paramètre $Q$ qui dépend de l'énergie électronique $\bar{w}$, et la densité électronique $n_{\mathrm{e}}$, qu'il faut déterminer.

III. Etude du gaz électronique dans la décharge. - L'excitation de la décharge est obtenue par couplage inductif. On peut lui appliquer la théorie développée par H. U. Eckert [9]. G'est une théorie de la colonne positive qui tient compte de la nonuniformité du champ électrique $E$ dans une section droite de la décharge du fait de son mode d'excitation.

1. Calgul des garagtéristiques de la décharge. - On peut ainsi calculer les grandeurs caractéristiques de la décharge : conductivité moyenne $\bar{\sigma}$, densité électronique moyenne $\bar{n}_{\mathrm{e}}$, énergie électronique 
moyenne $\bar{w}$ en fonction de la quantité $\frac{\nu\left|H_{R}\right|}{p^{2}}$ où $\left|H_{\mathrm{R}}\right|$ est le module du champ magnétique $H$ pour $r=R$. Ces relations dépendent du paramètre $p \times R$, où $p$ désigne la pression.

Connaissant la distribution du champ électrique $E$, on peut, de plus, calculer la puissance dissipée dans la décharge en fonction de $\frac{\nu\left|H_{\mathrm{R}}\right|}{p^{2}}$.

2. RÉGimes DE LA DÉGHARge. - Pour une valeur donnée du produit $p R$, il existe une valeur limite $\left[\frac{\nu\left|H_{\mathrm{R}}\right|}{p^{2}}\right]_{0}$ de la quantité $\frac{\nu^{\prime}\left|H_{\mathrm{R}}\right|}{p^{2}}$ qui annule $\bar{\sigma}$. La décharge ne peut donc pas s'entretenir pour une valeur de $\frac{\nu\left|H_{\mathrm{R}}\right|}{p^{2}}$ inférieure à cette limite.

Si $\bar{\sigma}$ est grande (épaisseur de peau du milieu conducteur inférieure au rayon de la décharge), toutes les grandeurs caractéristiques sont fixées de façon unique par la donnée de $\frac{\nu\left|H_{\mathrm{R}}\right|}{p^{2}}$, pour $p R$ donné, grâce aux résultats précédents.

Dans ce cas, la puissance dissipée par effet Joule peut être importante : plusieurs centaines de watts.

$\mathrm{Si} \bar{\sigma}$ n'est pas trop grande, les résultats du paragraphe précédent montrent que, pour $p R$ donné, la quantité $\frac{\nu\left|H_{\mathrm{R}}\right|}{p^{2}}$ reste constante et égale à la limite $\left[\frac{\nu\left|H_{R}\right|}{p^{2}}\right]_{0}$. Les différentes grandeurs, et $\bar{\sigma}$ en particulier, ne sont alors plus fixées par la donnée de $\left|H_{\mathrm{R}}\right|=\left|H_{\mathrm{R}}\right|_{0}$ seule et peuvent prendre une suite continue de valeurs. $\bar{\sigma}$ dépend alors du régime qui s'établit, caractérisé par la puissance dissipée.

On est dans ce cas si la puissance HF fournie est faible (de l'ordre de quelques dizaines de watts), ce qui se trouve réalisé dans les sources pour l'horloge à hydrogène. On étudiera ce cas en détail.

IV. Résultats numériques. - Ils sont rassemblés sur les figures 2 à 4 pour une décharge de $10 \mathrm{~cm}$ de longueur. La condition d'entretien de la décharge est caractérisée par les valeurs de $\left[\frac{\nu\left|H_{\mathrm{R}}\right|}{p^{2}}\right]_{0}$ qui sont fonction du produit $p R$ ( fig. 2). On voit que la décharge est plus difficile à établir dans un tube de petit rayon pour une pression donnée et que la condition d'entretien est d'autant plus facilement atteinte que la fréquence $\nu$ est plus élevée.

Les figures 3 et 4 montrent les variations de $m$ avec la puissance dissipée. On constate que $m$ atteint vite un palier vers la valeur 0,94. Quand la pression augmente, $m$ décroît, mais cette variation est moins rapide que celle de la pression, ce qui fait que le débit total d'atomes augmente avec la pression.

On constate enfin que la puissance nécessaire pour
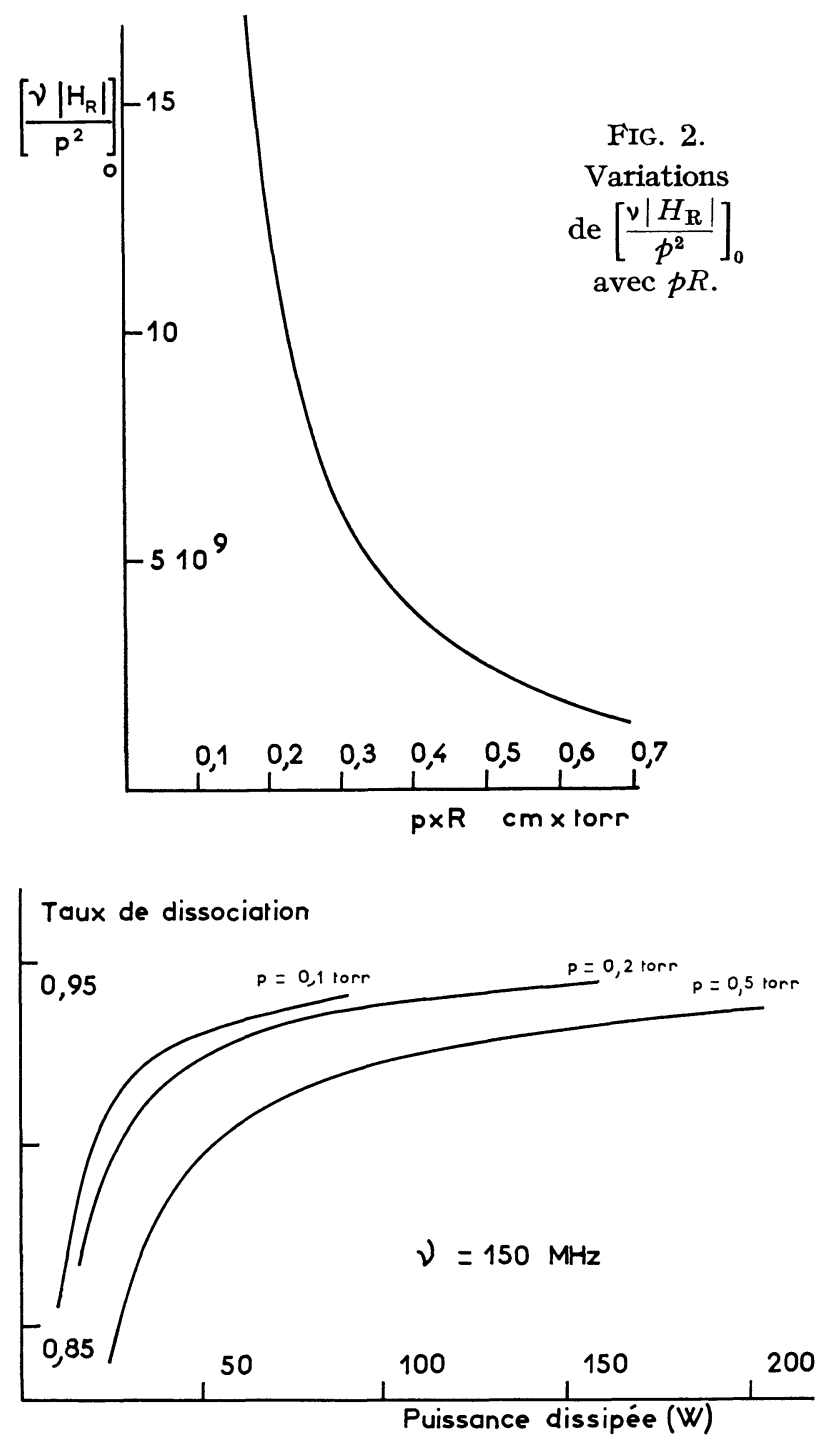

FIG. 3. - Variations de $m$ avec la puissance dissipée. Influence de la pression.

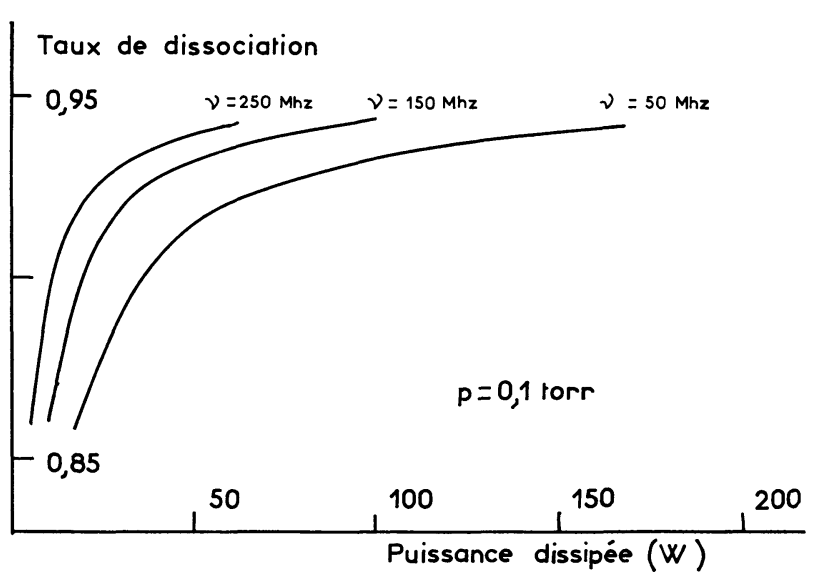

Frg. 4. - Variations de $m$ avec la puissance dissipée. Influence de la fréquence. 


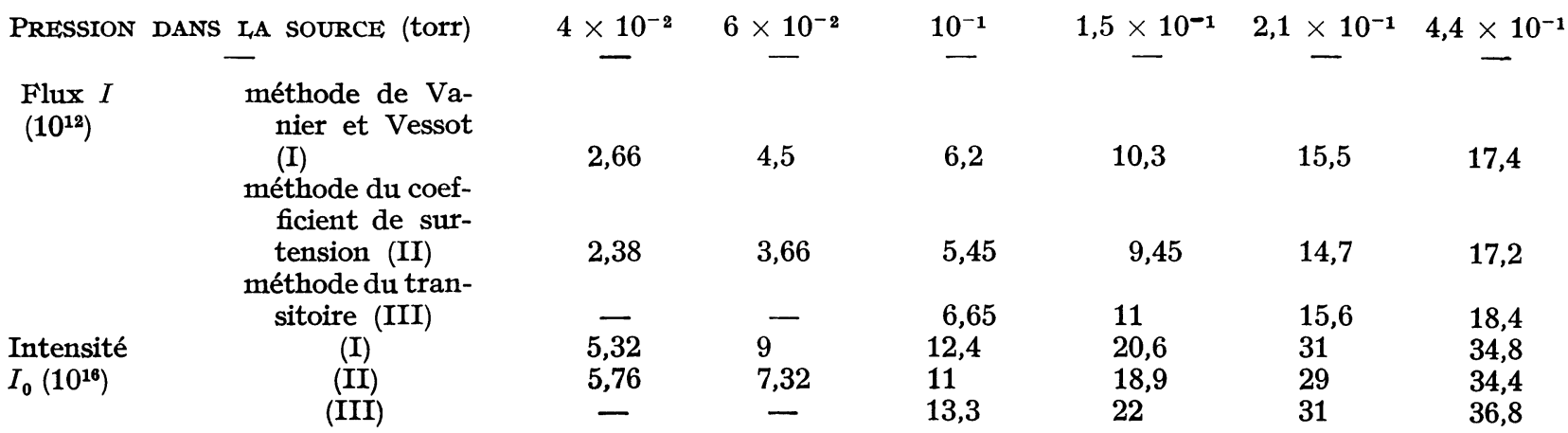

réaliser une valeur de $m$ donnée décroît quand la fréquence augmente, effet surtout sensible pour les valeurs élevées de $m$.

On voit donc l'intérêt qu'il y a à exciter la source à la fréquence la plus élevée possible.

V. Etude expérimentale. - Nous ne disposons actuellement que de résultats partiels, obtenus pour une seule gamme de fréquence d'excitation aux environs de $150 \mathrm{MHz}$.

1. Mesure diregte DE LA Goncentration en ATOMES $m$ A LA SORTIE DU TUBE DE DÉCHARGE. - On mesure la quantité de chaleur dégagée par la recombinaison des atomes du jet frappant une surface de cuivre.

Les résultats sont les suivants :

$\begin{array}{clll}p \text { (torr) } & 0,3 & 0,35 & 0,4 \\ m & 0,63 & 0,62 & 0,58\end{array}$

La fréquence d'excitation est de $125 \mathrm{MHz}$, on dissipe $50 \mathrm{~W}$ environ dans la décharge. On constate la diminution de $m$ avec la pression, prévue par la théorie.

2. MESURE INDIRECTE DU NOMBRE D'ATOMES D'HYDROGÈNE ÉMIS PAR LA SOURGE. - La source fournit des atomes $H$ qui alimentent une horloge à hydrogène [10] . Connaissant les caractéristiques du système de focalisation [10], on peut calculer la trajectoire d'un atome issu d'un point quelconque de la source [11]. Si on suppose que les vitesses sont réparties dans le jet selon une loi de Maxwell correspondant à une température dans la source de $T=323^{\circ} \mathrm{K}$, on peut relier le flux entrant dans la cavité du maser, $I$, à l'intensité axiale $I_{0}$ du jet issu de la source.

On trouve, dans les conditions réalisées :

$$
I=5 \times 10^{-5} I_{0} \text {. }
$$

Dans cette relation, $I$ représente le flux d'atomes entrant dans l'état $F=1, m=0$ et $I_{0}$ l'intensité axiale totale des atomes dans tous les états.

D'autre part, $I$ peut être déterminée par l'étude des constantes de temps $T_{1}$ et $T_{2}$ caractéristiques du maser [12].

On a donc les valeurs mesurées de $I$ et de $I_{0}$ (voir tableau ci-dessus).

A partir des calculs de la référence [13] et en tenant compte du fait que les atomes sont soumis dans la source aux chocs contre un autre atome ou contre une molécule, on peut calculer cette intensité axiale $I_{0}$ en fonction de la pression dans la source et de $m$.

La comparaison aux valeurs mesurées conduit aux valeurs suivantes du rapport $m$ :

$\begin{array}{cccc}p \text { (torr) } & 0,04 & 0,06 & 0,1 \\ m & 0,49-0,51 & 0,50-0,56 & 0,53-0,58 \\ p \text { (torr) } & 0,15 & 0,21 & 0,44 \\ m & 0,62-0,66 & 0,68-0,70 & 0,63-0,65\end{array}$

On a obtenu des résultats cohérents qui peuvent être repris de façon systématique. Il est donc possible, par ce procédé, d'étudier expérimentalement la production des atomes polarisés d'une source de protons polarisés.

Manuscrit reçu le 10 mai 1967.

\section{BIBLIOGRAPHIE}

[1] Luccio (A.), Merzagora (N.), Succy (C.), Nuovo Cimento, 1964, 33, 1710 .

[2] Colinss (E. R.), Gavish (H. F.), Whineray (S.), Nuclear Instruments and Methods, 1963, 25, 67.

[3] BeURTeY (R.), PAPINEAU (A.), Thirion (J.), Nuovo Cimento, 1961, 19, Supplemento, 207.

[4] Goodyear (C. C.), von Enget, (A.), Proc. Phys. Society, 1962, 79, 734

[5] KELIER (R.), DICK (L.), FIDECARo (M.), Rapport C.E.R.N., 1960, 60, 2

[6] Smith (W. V.), J. Chemical Physics, 1943, 111, 110.
[7] Steiner (R. O.), Trans. Favaday Society, 1935, 31, 623.

[8] Admur (I.), J. Chemical Physics, 1936, 4, 339.

[9] ECKERT (H. U.), J. Applied Physics, 1962, 33, 2780.

[10] DesaintFuscien (M.), Schermann (J. P.), Onde électrique, octobre 1966.

[11] Audoin (C.), Schermann (J. P.), C. R. Acad. Sci., 1967, 264, 647.

[12] Audoin (C.), DesainTFuscien (M.), SchermanN (J. P.), C. R. Acad. Sci., 1967, 264, 698.

[13] GiordmaIne (J. A.), Wang (T. C.), J. Applied Physics, 1960, 31, 463. 